\title{
Does Adductor Canal Block Have a Synergistic Effect with Local Infiltration Analgesia for Enhancing Ambulation and Improving Analgesia after Total Knee Arthroplasty?
}

Wirinaree Kampitak, $\mathrm{MD}^{1}$, Aree Tanavalee, $\mathrm{MD}^{2}$, Srihatach Ngarmukos, $\mathrm{MD}^{2}$, Chavarin Amarase, $\mathrm{MD}^{2}$, Rawiwan Apihansakorn, $\mathrm{MD}^{1}$, and Pannika Vorapalux, $\mathrm{MD}^{1}$

Departments of ${ }^{1}$ Anesthesiology and ${ }^{2}$ Orthopaedics, King Chulalongkorn Memorial Hospital and Faculty of Medicine, Chulalongkorn University, Bangkok, Thailand

\begin{abstract}
Purpose: We compared a single-injection adductor canal block (ACB) with or without local infiltration analgesia (LIA) for accelerating functional recovery and reducing postoperative pain after total knee arthroplasty (TKA).

Materials and Methods: Sixty-two patients undergoing TKA with simple spinal analgesia and ACB were randomized to receive either LIA (group $\mathrm{A}+\mathrm{L}$ ) or placebo LIA (group A). Postoperative visual analog scale (VAS) score for pain, Timed Up and Go (TUG) test and quadriceps strength, total dosage of rescue analgesia, time to first rescue analgesia, and adverse events were serially evaluated from postoperative day 1 to 3 months.

Results: There were no differences between both groups in pre- and postoperative VAS, TUG test, quadriceps strength 2 days, 3 days, 2 weeks, 6 weeks, and 3 months postoperatively. There were no differences in Knee Society clinical and function scores at 6 months and 1 year. However, group $\mathrm{A}+\mathrm{L}$ had a significantly longer time for postoperative rescue analgesia (491 minutes vs. 143 minutes, $\mathrm{p}=0.04)$ with less patients requiring rescue analgesia during 6 hours after surgery $(16.7 \%$ vs. $43.3 \%, \mathrm{p}=0.024)$. Both groups had similarly high rates of patient satisfaction with low adverse event rates.

Conclusions: Combined ACB and LIA in TKA enhanced early ambulation with reduced and delayed rescue analgesia.
\end{abstract}

Keywords: Knee, Arthroplasty, Adductor canal block, Local analgesia, Pain, Ambulation

\section{Introduction}

Total knee arthroplasty (TKA) has been associated with severe postoperative pain, especially during the first 48 hours after sur-

Received November 14, 2017; Revised November 27, 2017;

Accepted March 9, 2018

Correspondence to: Aree Tanavalee, MD

Department of Orthopaedics, King Chulalongkorn Memorial Hospital and Faculty of Medicine, Chulalongkorn University, 1873 Rama 4 Road, Pathumwan, Bangkok 10330, Thailand

Tel: +66-22564212, Fax: +66-22564625

E-mail: areetana@hotmail.com

Source of funding: This research was supported by the Ratchadapiseksompotch Fund, Faculty of Medicine, Chulalongkorn University (IRB No. 601/57).

This is an Open Access article distributed under the terms of the Creative Commons Attribution Non-Commercial License (http://creativecommons.org/licenses/by-nc/4.0/) which permits unrestricted non-commercial use, distribution, and reproduction in any medium, provided the original work is properly cited. gery ${ }^{1,2)}$. Several multimodal analgesic regimens including peripheral nerve blocks have been proposed for effective postoperative pain control after $\mathrm{TKA}^{3-6)}$. Although femoral nerve block (FNB), a widely used peripheral nerve block, provided a very satisfied postoperative pain control, it could accentuate quadriceps muscle weakness and contribute to a higher rate of falls at early ambulation $^{7}$. Therefore, the optimal multimodal analgesic regimens following TKA should offer a well balance between adequate pain control and sufficient lower motor function, especially preserved quadriceps strength to allow for safe early ambulation.

Currently, two methods of multimodal analgesia have become popular. The adductor canal block (ACB), a blockade of the saphenous nerve and/ or the cutaneous branches of the femoral nerve in the adductor canal, has been proposed as an optional peripheral nerve block in multimodal analgesic regimen ${ }^{8-11)}$ with its superiority to FNB, in terms of negative effect on quadriceps strength. However, it innervated nerves that serve sensory func- 


\section{Kampitak et al. Synergistic Effect of Adductor Canal Block and Local Analgesia after TKA}

tion in only the anterior part of the knee capsule ${ }^{12,13)}$. The local infiltration analgesia (LIA), has been introduced to become a major part of pain control regimens for TKA due to its simplicity and apparent safety. However, rebound and severe postoperative analgesia after LIA effect wears off has been observed; therefore, it may not be an effective single analgesia ${ }^{14-19)}$. The recent studies demonstrated that the addition of LIA to peripheral nerve block provided better pain relief and less opioid consumption after TKA than peripheral nerve block alone or LIA alone ${ }^{20-22)}$. However, the clinical advantage of LIA in supplement to peripheral nerve block, especially ACB, remains unclear.

The objective of the present study was to determine the benefit of the addition of single- dose LIA to a single-injection ACB compared with ACB alone on functional ability and pain after TKA. We hypothesized that there would be no difference between the combination of ACB with single-dose LIA and single-injection ACB alone with regard to accelerating ambulation ability and improving postoperative pain after TKA.

\section{Materials and Methods}

\section{Study Design}

This single-center, randomized, placebo-controlled, doubleblind trial study was approved by the Institutional Review Board and was registered with Clinicaltrials.in.th, identifier TCTR20150720006. From April 2015 to April 2016, 71 patients scheduled to undergo uncomplicated unilateral TKA were enrolled in this study. Inclusion criteria were patients aged more than 18 years with the American Society of Anesthesiologists (ASA) physical status I to III. Exclusion criteria were contraindication for neuraxial and/or regional anesthesia, history of allergy to drugs implicated in this study, chronic pain requiring opioid medication, neuropathic pain, failure in the preoperative Timed Up and Go (TUG) test, and subject refusal.

All patients were randomized to receive either a combined $\mathrm{ACB}$ and LIA mixture (group $\mathrm{A}+\mathrm{L}$ ) or $\mathrm{ACB}$ and $0.9 \%$ saline as a placebo (group A) using a computer generated random number table. The concealed assignments were opened in the operation room and one uninvolved anesthesiologist was assigned to prepare the LIA mixture and placebo. All patients were scheduled to undergo surgery in the morning as the first case of the day under a simple spinal analgesia using $0.5 \%$ hyperbaric bupivacaine $3 \mathrm{~mL}$. All surgeries were performed by 2 senior surgeons using a single surgical technique (mini-midvastus approach) with a tourniquet pressure of $320 \mathrm{mmHg}$. A single total knee prosthesis, NexGen posterior stabilized knee system (NexGen-LPS; Zimmer, Warsaw,
IN, USA), was used in all knees. After surgery, the tourniquet was not deflated for hemostasis. The vacuum drain was inserted and removed 18-22 hours after surgery.

Before wound closure, both senior surgeons who performed surgeries were blinded to injection of LIA mixture or placebo. The LIA mixture, which contained the same concentration of morphine according to the first study using LIA in TKA of Busch et al. ${ }^{16)}$ (0.5\% levobupivacaine $20 \mathrm{~mL}$, morphine $5 \mathrm{mg}$, adrenaline $0.3 \mathrm{mg}$ in saline solution in a total volume of $100 \mathrm{~mL}$ ), was injected into synovial tissue and joint capsule around the knee joint. Then, a single-dose ACB was performed in all patients at the recovery unit. After identification of the adductor canal at the midthigh level using a high-frequency linear ultrasound transducer (10-12 Hz; SonoSite Turbo, SonoSite Inc., Bothell, WA, USA), a total of $0.5 \%$ levobupivacaine $20 \mathrm{~mL}$ was injected into the adductor canal by using a 22-guage, $100 \mathrm{~mm}$ needle (Stimuplex; B Braun, Bethlehem, PA, USA). All patients received a premedication of acetaminophen $650 \mathrm{mg}$ at 30 minutes before surgery. Dexamethasone $10 \mathrm{mg}$ and ondansetron $4 \mathrm{mg}$ were administered intravenously in all patients for postoperative nausea and vomiting prophylaxis.

Regarding pain rescue, all patients received a patient-controlled analgesia (PCA) device for 48 hours postoperatively, with morphine $2 \mathrm{mg} /$ dose, 10-minute lockout time and no basal infusion. In all patients, intravenous parecoxib (Dynastat) $40 \mathrm{mg}$ was administered every 12 hours for 3 consecutive doses starting at the recovery unit, and oral daily celecoxib (Celebrex) $400 \mathrm{mg}$ was continued after the last dose of parecoxib. Oral Acetaminophen $650 \mathrm{mg}$ every 6 hours was started at the patient ward for 1 day. Daily esomeprazole (Nexium) $40 \mathrm{mg}$ was started from the night of postoperative day 0. Daily pregabalin (Lyrica) $75 \mathrm{mg}$ was started from the night of postoperative day 1 . Metoclopramide $5 \mathrm{mg}$ was administered intravenously for rescuing nausea and vomiting and chlorpheniramine $5 \mathrm{mg}$ was administered intravenously to reduce itching.

\section{Outcome Measures}

Preoperatively, patients were instructed for pre- and postoperative ambulation ability assessment, quadriceps strength test and visual analog scale (VAS) score. Demographic characteristics, preoperative VAS score, TUG test and quadriceps strength were serially recorded. The TUG test was defined by measuring the time to rise from an arm chair (seat height at $46 \mathrm{~cm}$ ), walk for $3 \mathrm{~m}$, turn, and return to sitting in the same chair without physical assistance $^{23)}$. Quadriceps strength test was performed according to the method of Judd et al. ${ }^{24)}$ using an isometric force dynamom- 
eter (MicroFET2; Hoggan Scientific, Salt Lake City, UT, USA).

From the morning of postoperative day 1 from 7 to 8 am, all patients were encouraged to sit, straighten both legs, stand up and walk using a walker as tolerated. All patients were allowed to ambulate independently with walking aid until discharge. Discharge criteria included stable vital signs, good appetite, no local wound problems, full weight bearing on the operated limb, and $90^{\circ}$ knee flexion. All patients were prescribed oral medications, including ultracet 0.5 tab twice a day, daily celecoxib $200 \mathrm{mg}$, daily pregabalin $75 \mathrm{mg}$, and daily esomeprazole $20 \mathrm{mg}$ for 2 weeks. Tramadol $50 \mathrm{mg}$ was prescribed for pain rescue as needed every 6 hours.

A serial evaluation of postoperative pain was conducted at rest and at movement using VAS from postoperative day 1 to day 3 , and then at 2 weeks, 6 weeks, and 3 months by an independent evaluator. The Knee Society System (KSS) clinical and function scores of both groups were evaluated at 6 months and 1 year.

The morphine consumptions via PCA device were recorded for the first time of requirement and at 3, 12, 24 and 48 hours postoperatively. Similarly, the TUG test and the quadriceps strength test were also serially recorded by an independent physiotherapist.

\section{Sample Size and Statistical Analysis}

In a pilot study of 10 patients who received spinal anesthesia combined with ACB alone, the mean time for TUG test on postoperative day 2 was $92.5 \pm 26.5$ seconds. We assumed that a $25 \%$ reduction should be considered clinically significant. For a non-inferiority or superiority trial with continuous outcome, we calculated that 28 patients in each group were required to detect a mean difference of 23.1 seconds with standard deviation of 26.4 seconds on the TUG test using the $t$-test (independent samples), $\alpha=0.05$ and a power of $80 \%$. We planned for an inclusion of 31 patients to compensate for dropouts.

Statistical analysis was performed by using IBM SPSS ver. 22.0 (IBM Corp., Armonk, NY, USA). Repeated measures analysis of variance were used for the analysis of TUG test and VAS. Categorical data were analyzed by using the chi-square test or Fisher exact test. Normal distributed data were statistically tested with the independent $t$-test, and data that did not fulfill the assumptions of normal distribution were analyzed with the MannWhitney $U$-test. The paired $t$-test was used to compare a withinsubjects test group before and after an intervention. A p-value of less than 0.05 was considered to be statistically significant.

\section{Results}

Patient recruitment and flow through the protocol are described

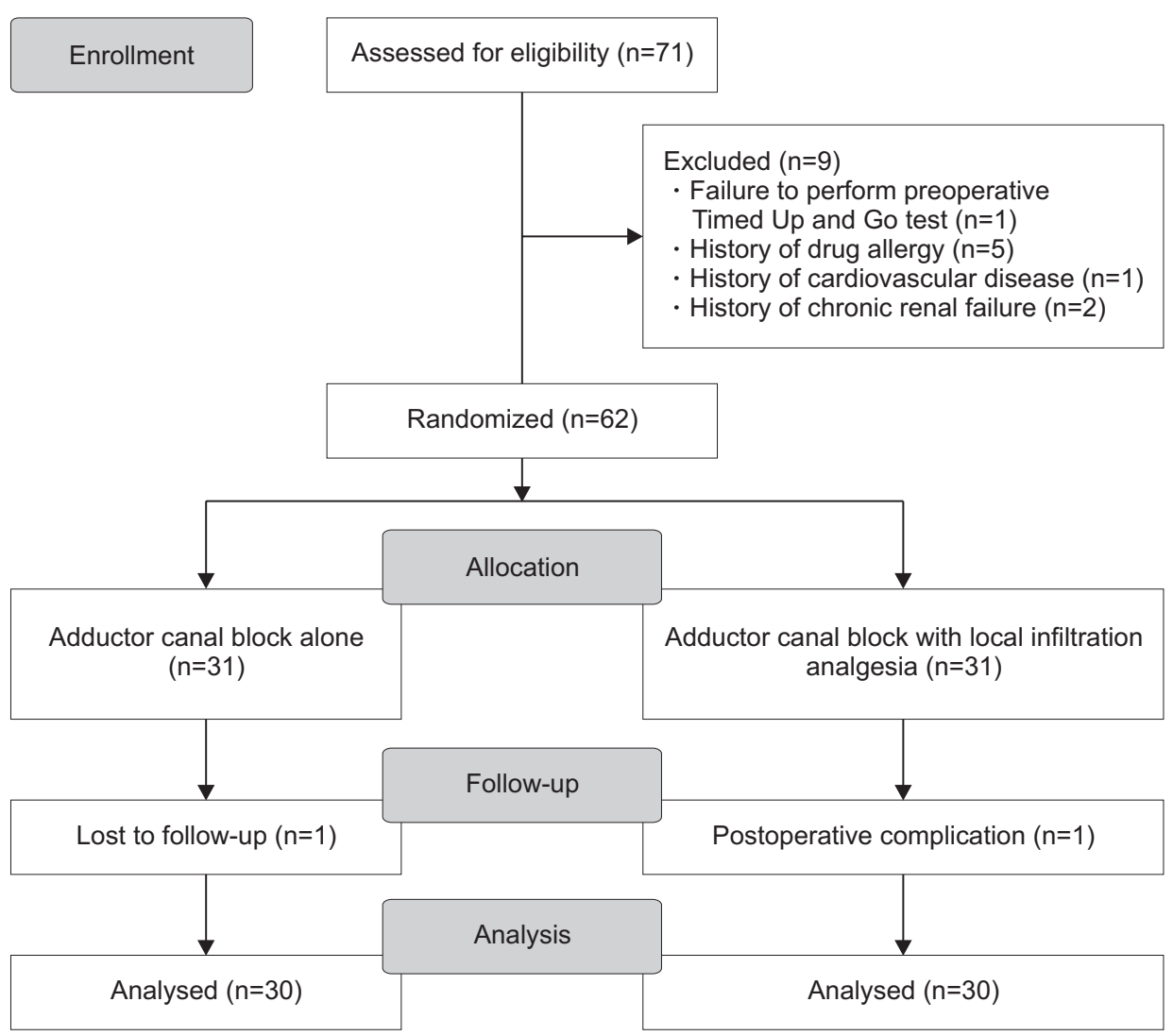

Fig. 1. Consolidated Standards of Reporting Trials (CONSORT) flow diagram. 


\section{Kampitak et al. Synergistic Effect of Adductor Canal Block and Local Analgesia after TKA}

in the Consolidated Standards of Reporting Trials (CONSORT) diagram (Fig. 1). Nine patients were excluded from the study due to patient's impaired compliance and disagreement to continue. Therefore, 62 patients were randomized and evaluated. At early follow-up, 60 patients ( 30 in group $\mathrm{A}+\mathrm{L}$ and 30 in group $\mathrm{A}$ ) were available for complete evaluation; however, at 1-year follow-up, 2 patients in group $\mathrm{A}+\mathrm{L}$ and 1 patient in group $\mathrm{A}$ were not able for clinical evaluation. Demographic data, duration of surgery, preoperative TUG test, preoperative quadriceps strength, and length of hospital stay of both groups were not significantly different (Table 1).

There were no differences of postoperative VAS on postoperative day 1 to day 3 and at all points of follow-up between both groups as shown in Table 2. The median time to request the first analgesic was significantly longer in group $\mathrm{A}+\mathrm{L}$ than group $\mathrm{A}$ with a significantly lower percentage of patients requiring rescue analgesia at a duration of 6 hours after surgery. However, at a

Table 1. Patient Characteristics

\begin{tabular}{|c|c|c|c|}
\hline Parameter & Group A $(n=30)$ & Group $A+L(n=30)$ & $\mathrm{p}$-value \\
\hline Age (yr) & $72.37 \pm 8.02$ & $69.1 \pm 5.36$ & 0.069 \\
\hline \multicolumn{4}{|l|}{ Gender } \\
\hline Male & $3(10)$ & $4(13.3)$ & 1 \\
\hline Female & $27(90)$ & $26(86.7)$ & \\
\hline Body mass index $\left(\mathrm{kg} / \mathrm{m}^{2}\right)$ & $26.25 \pm 3.72$ & $27.92 \pm 4.64$ & 0.13 \\
\hline \multicolumn{4}{|l|}{ ASA classification } \\
\hline ASA 1 & $0(0)$ & $0(0)$ & 1 \\
\hline ASA 2 & $29(96.7)$ & $28(93.3)$ & \\
\hline ASA 3 & $1(3.3)$ & $2(6.7)$ & \\
\hline ASA 4 & $0(0)$ & $0(0)$ & \\
\hline \multicolumn{4}{|l|}{ Side } \\
\hline Left & $16(53.3)$ & $16(53.3)$ & 1 \\
\hline Right & $14(46.7)$ & $14(46.7)$ & \\
\hline \multicolumn{4}{|l|}{ Preoperative evaluation } \\
\hline VAS at rest & $3.2 \pm 2.1$ & $3.6 \pm 2.0$ & 0.48 \\
\hline VAS at movement & $7.4 \pm 2.4$ & $7.5 \pm 2.5$ & 0.67 \\
\hline \multicolumn{4}{|l|}{ Quadriceps strength (N/m²) } \\
\hline Knee full extension & $98.41 \pm 43.11$ & $104.02 \pm 42.31$ & 0.61 \\
\hline $45^{\circ}$ flexion & $129.56 \pm 61.99$ & $125.31 \pm 54.95$ & 0.78 \\
\hline $90^{\circ}$ flexion & $119.72 \pm 56.36$ & $120.05 \pm 53.23$ & 0.98 \\
\hline Surgical time (min) & $119.47 \pm 25.76$ & $112.83 \pm 28$ & 0.34 \\
\hline Hospital stay (day) & $4 \pm 0$ & $4 \pm 0$ & 1 \\
\hline
\end{tabular}

Values are presented as mean \pm standard deviation or number (\%). Group A: adductor canal block group, Group A+L: adductor canal block combined with local infiltration analgesia group, ASA: American Society of Anesthesiologists, VAS: visual analog scale. duration of 12 hours after surgery, both groups had similar percentages of patients requiring rescue analgesia, and the overall morphine consumption of both groups at 24 and 48 hours were

Table 2. Visual Analog Scale (VAS) from Postoperative Day 0 to Day 3

\begin{tabular}{llll}
\hline \multicolumn{1}{c}{ Parameter } & Group A & Group A+L & p-value \\
\hline VAS POD 0 & & & \\
6 hours & $1.94 \pm 1.74$ & $1.23 \pm 1.17$ & 0.07 \\
12 hours & $1.29 \pm 2.03$ & $0.84 \pm 0.85$ & 0.28 \\
18 hours & $1.46 \pm 1.7$ & $1.45 \pm 1.3$ & 0.97 \\
VAS POD 1 at 8 am & & & \\
Rest & $1.49 \pm 1.60$ & $1.05 \pm 1.02$ & 0.21 \\
Sit & $1.88 \pm 1.64$ & $1.78 \pm 1.15$ & 0.79 \\
Knee flexion & $2.89 \pm 1.59$ & $2.43 \pm 1.42$ & 0.25 \\
Knee extension & $2.36 \pm 1.76$ & $2.26 \pm 1.26$ & 0.80 \\
VAS POD 2 at 8 am & & & \\
Rest & $1.23 \pm 1.30$ & $1.94 \pm 1.51$ & 0.06 \\
Sit & $1.93 \pm 1.75$ & $2.50 \pm 1.18$ & 0.14 \\
Stand & $2.50 \pm 2.01$ & $3.24 \pm 1.31$ & 0.10 \\
Walk & $2.58 \pm 1.8$ & $3.27 \pm 1.46$ & 0.11 \\
VAS POD 3 at 8 am & & & \\
Rest & $1.36 \pm 1.34$ & $1.65 \pm 1.26$ & 0.38 \\
Sit & $1.66 \pm 1.45$ & $2.15 \pm 1.32$ & 0.17 \\
Stand & $1.94 \pm 1.36$ & $2.62 \pm 1.71$ & 0.09 \\
Walk & $2.22 \pm 1.36$ & $2.55 \pm 1.65$ & 0.40 \\
\hline
\end{tabular}

Values are presented as mean \pm standard deviation.

Group A: adductor canal block group, Group A+L: adductor canal block combined with local infiltration analgesia group, POD: postoperative day.

Table 3. Time to First Request for Rescue Analgesia and Morphine Consumption

\begin{tabular}{lccc}
\hline \multicolumn{1}{c}{ Parameter } & $\begin{array}{c}\text { Group A } \\
(\mathrm{n}=30)\end{array}$ & $\begin{array}{c}\text { Group A+L } \\
(\mathrm{n}=30)\end{array}$ & p-value \\
\hline $\begin{array}{l}\text { Duration to the 1st morphine } \\
\text { request (min) }\end{array}$ & 143 & 490.5 & 0.040 \\
$\quad$ Shortest time & 105 & 150 & \\
$\quad$ Longest time & 498 & 1,090 & \\
Patients who requested morphine, no. (\%) & & \\
At $\leq 6$ hours & $13(43.3)$ & $5(16.7)$ & 0.024 \\
Morphine consumption (mg), mean (range) & & \\
Up to 12 hours & $4(0-8)$ & $2(0-4)$ & 0.15 \\
Up to 24 hours & $6(0-14)$ & $4(0-8)$ & 0.30 \\
Up to 47 hours & $10(4-20)$ & $9(4-18)$ & 0.84 \\
\hline
\end{tabular}

Group A: adductor canal block group, Group A+L: adductor canal block combined with local infiltration analgesia group. 
Table 4. Adverse Events from Postoperative Day 1 to Day 3

\begin{tabular}{|c|c|c|c|}
\hline Parameter & $\begin{array}{c}\text { Group A } \\
(\mathrm{n}=30)\end{array}$ & $\begin{array}{c}\text { Group A+L } \\
(\mathrm{n}=30)\end{array}$ & p-value \\
\hline Overall fall & $0(0)$ & $0(0)$ & 1.00 \\
\hline \multicolumn{4}{|l|}{ Nausia and vomiting } \\
\hline \multicolumn{4}{|l|}{ POD 1} \\
\hline None & $24(80)$ & $17(56.7)$ & 0.06 \\
\hline Queasy & $3(10)$ & $6(20)$ & \\
\hline Severe nausea & $0(0)$ & $5(16.7)$ & \\
\hline Vomiting & $3(10)$ & $2(6.7)$ & \\
\hline \multicolumn{4}{|l|}{ POD 2} \\
\hline None & $24(80)$ & $23(76.7)$ & 0.95 \\
\hline Queasy & $5(16.7)$ & $6(20)$ & \\
\hline Severe nausea & $1(3.3)$ & $1(3.3)$ & \\
\hline Vomiting & $0(0)$ & $0(0)$ & \\
\hline \multicolumn{4}{|l|}{ POD 3} \\
\hline None & $26(86.7)$ & $19(63.3)$ & 0.13 \\
\hline Queasy & $2(6.7)$ & $8(26.7)$ & \\
\hline Severe nausea & $0(0)$ & $1(3.3)$ & \\
\hline Vomiting & $0(0)$ & $0(0)$ & \\
\hline \multicolumn{4}{|l|}{ Pruritus } \\
\hline \multicolumn{4}{|l|}{ POD 1} \\
\hline None & $22(73.3)$ & $19(63.3)$ & 0.10 \\
\hline Mild \& no treatment & $5(16.7)$ & $9(30)$ & \\
\hline Moderate \& treatment & $3(10)$ & $0(0)$ & \\
\hline Severe \& treatment & $0(0)$ & $2(6.7)$ & \\
\hline \multicolumn{4}{|l|}{ POD 2} \\
\hline None & $21(70)$ & $25(83.3)$ & 0.20 \\
\hline Mild \& no treatment & $9(30)$ & $4(13.3)$ & \\
\hline Moderate \& treatment & $0(0)$ & $1(3.3)$ & \\
\hline Severe \& treatment & $0(0)$ & $0(0)$ & \\
\hline \multicolumn{4}{|l|}{ POD 3} \\
\hline None & $27(90)$ & $28(93.3)$ & 1.00 \\
\hline Mild \& no treatment & $3(10)$ & $2(6.7)$ & \\
\hline Moderate \& treatment & $0(0)$ & $0(0)$ & \\
\hline Severe \& treatment & $0(0)$ & $0(0)$ & \\
\hline
\end{tabular}

Values are presented as number (\%).

Group A: adductor canal block group, Group A+L: adductor canal block combined with local infiltration analgesia group, POD: postoperative day.

not significantly different (Table 3). There were similarly low incidences of nausea/vomiting and pruritus between groups (Table 4). No patient's falls were recorded in either arm during the study period.

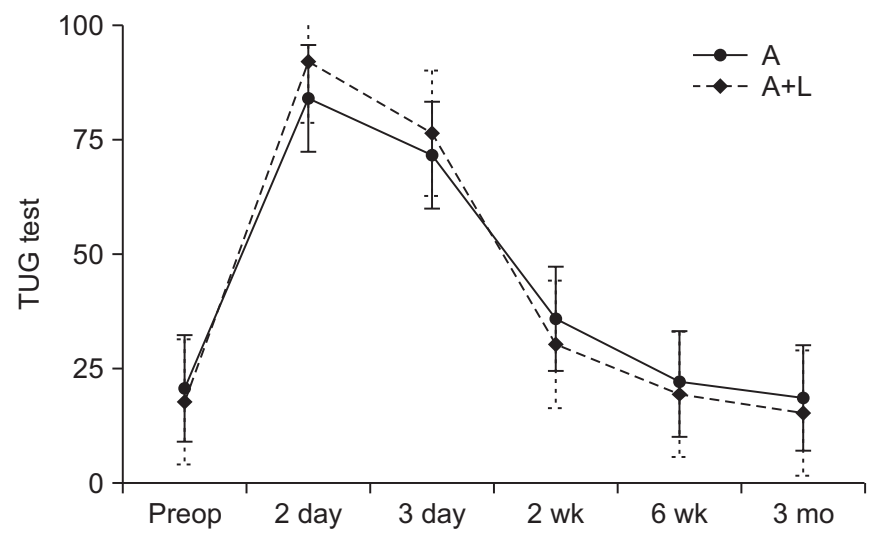

Fig. 2. Timed Up and Go (TUG) test results on pre- and postoperative evaluation at different points of follow-up reported as mean \pm standard deviation. There were no significant differences between the groups at all points of follow-up. Value of TUG test is shown in seconds. A: adductor canal block group, $\mathrm{A}+\mathrm{L}$ : adductor canal block combined with local infiltration analgesia group, Preop: preoperative.

There were no differences in TUG test between both groups at all points of follow-up as shown in Fig. 2. However, in comparison to the preoperative evaluation, both groups had significantly extended time of postoperative TUG test, which returned to a similar value of the preoperative period at 6-week follow-up. The postoperative quadriceps strengths during knee full extension, $45^{\circ}$ flexion, and $90^{\circ}$ flexion on postoperative days 2 and 3 were similar between both groups at all points of follow-up as shown in Fig. 3. The overall patient satisfaction rates of group $\mathrm{A}+\mathrm{L}$ and group A were $89 \%$ and $88 \%$, respectively, showing no intergroup difference. There were differences in KSS clinical and function scores at 6 months and 1 year of follow-up as shown in Table 5. There was no readmission or no revision for any reasons in this study.

\section{Discussion}

The present randomized, placebo-controlled study was conducted to evaluate the benefit of the addition of LIA to a singledose ACB compared to single-dose ACB alone on postoperative pain, early ambulation ability, and quadriceps strength after TKA, which resulted in similar VAS, TUG test, and quadriceps strength at all points of measurement, as well as similarly low total morphine consumption and adverse reactions and high patient satisfaction rates in both groups. These findings imply that both regimens of multimodal pain control were similarly effective without interference of quadriceps strength which was found related to $\mathrm{FNB}^{7)}$.

Significant advantages of combined LIA with single-dose ACB 

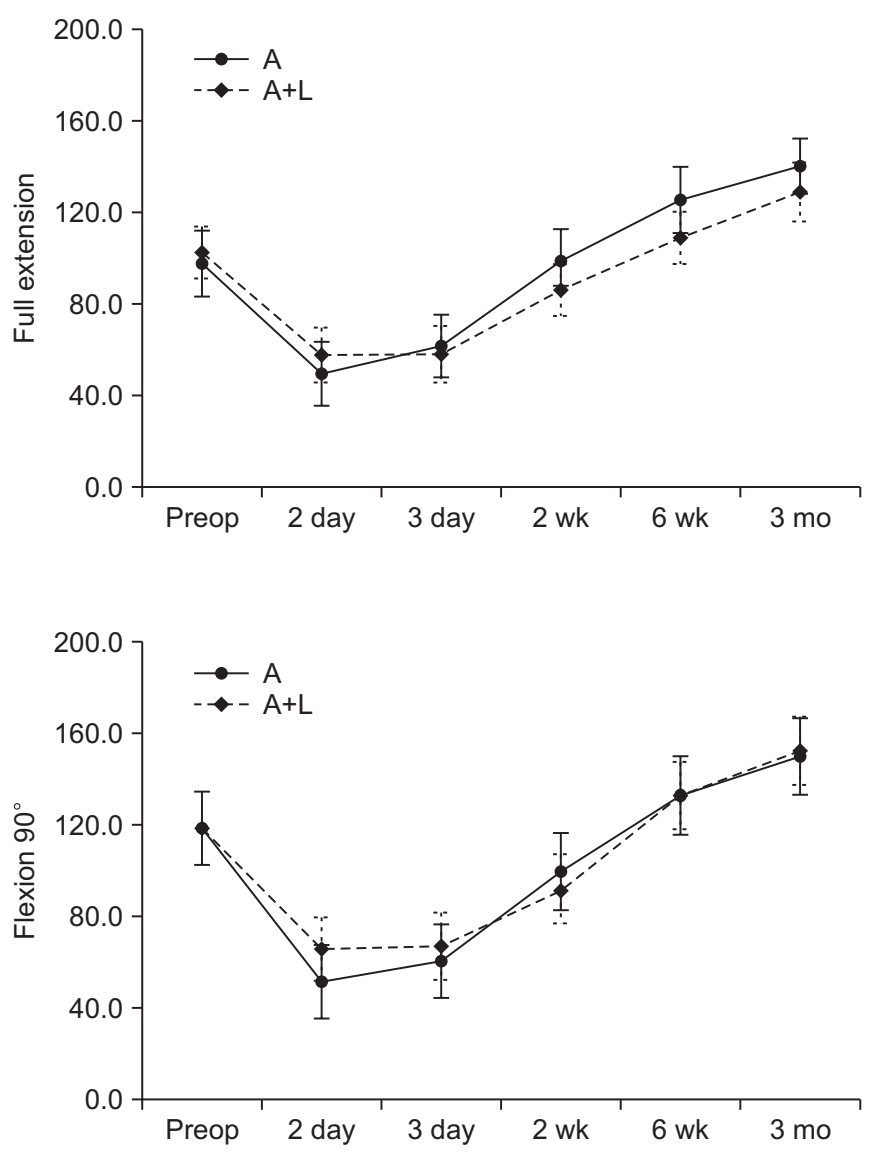

Table 5. KSS Clinical and Function Scores

\begin{tabular}{lccc}
\hline \multicolumn{1}{c}{ Parameter } & Group A & Group A+L & p-value \\
\hline Follow-up (mo) & 15.3 & 15.7 & $>0.05$ \\
Preop KSS clinical score (point) & $34.3(30)$ & $34.7(30)$ & $>0.05$ \\
Preop KSS function score (point) & $32.2(30)$ & $31.8(30)$ & $>0.05$ \\
Six-month follow-up (point) & & & \\
KSS clinical score & $84.3(29)$ & $84.5(30)$ & $>0.05$ \\
KSS function score & $72.9(29)$ & $72.8(30)$ & $>0.05$ \\
One-year follow-up (point) & & & \\
KSS clinical score & $92.1(29)$ & $92.9(28)$ & $>0.05$ \\
KSS function score & $89.7(29)$ & $89.5(28)$ & $>0.05$ \\
\hline
\end{tabular}

Values are presented as mean (number).

Group A: adductor canal block group, Group A+L: adductor canal block combined with local infiltration analgesia group, Preop: preoperative, KSS: Knee Society System.

included delaying the time for the first request of rescue analgesia with less patients requiring rescue analgesia during 6 postoperative hours. These results might be due to the ability of the LIA to provide local analgesia into the entire area of soft tissue around the surgical site, which could contribute to greater analgesia

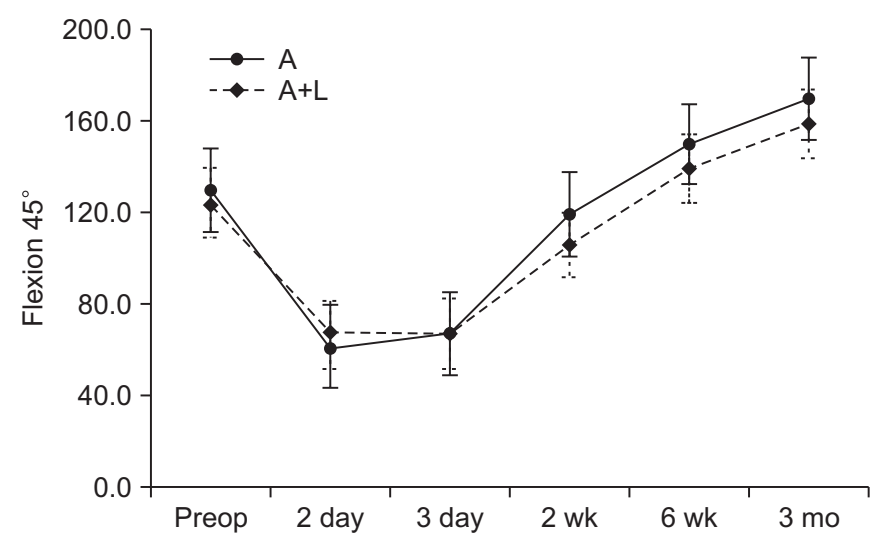

Fig. 3. Quadriceps strength with the knee in full extension, $45^{\circ}$ flexion, and $90^{\circ}$ flexion on pre- and postoperative evaluation at different points of follow-up reported as mean \pm standard deviation. There were no significant differences between the groups at all points of follow-up. Value of quadriceps strength is shown in $\mathrm{N} / \mathrm{m}$. A: adductor canal block group, A+L: adductor canal block combined with local infiltration analgesia group, Preop: preoperative.

than only the use of ACB. However, the effect of the combination treatment may have not persisted longer than 6-8 hours postoperatively after TKA. These results are consistent with prior studies $^{9,17)}$ that showed the duration of the ACB (20-22 hours) was longer than the LIA (6-12 hours) for postoperative analgesia. Therefore, there was no difference in pain score and morphine consumption after 12 hours postoperatively between groups in this study. In addition, the primary outcome (TUG test on postoperative day 2) was not significantly different between the two groups. It might possibly be due to several reasons including very low levels of postoperative pain after TKA, resulting in no or minimal effects on pain on ambulation in both groups, as well as inter- or intrapersonal variabilities such as physiotherapistpatient coordination or patient anxiety when TUG test was measured.

Disadvantages of these combined LIA and single-dose ACB can be described as technically demanding and time consuming. To improve the efficiency of these combined procedures, the anesthesiologist should have adequate practice in order to develop consistent skills to perform a reliable ACB under ultrasound guidance in limited time.

The results of the present study disagree with the studies of Koh 
et $\mathrm{al}^{20)}$ and Sawhney et al. ${ }^{25)}$. These studies reported superior analgesic effect of combined ACB and LIA than ACB alone or LIA alone on postoperative mobility after TKA. Contrary to their study which used nonsteroidal anti-inflammatory drug (NSAID) only in the LIA group, the present study used NSAID in both groups which might be related to the similarly satisfactory pain control and very low total morphine consumption for rescue analgesia in both groups. A recent study has shown that NSAID adjuvants in LIA mixture or systemic administration enhanced less opioid consumption and provided earlier mobilization and hospital discharge than those methods without NSAID ${ }^{26}$. Therefore, the positive analgesic effect of parecoxib in the present study might have played a role in multimodal pain control, minimizing the total morphine consumption in both groups.

The assessment of the efficacy of the LIA and the ACB might be obscured by the effect of spinal analgesia. However, spinal analgesia in the present study was designed to use only bupivacaine. Therefore, its effect generally does not persist more than 1-1.5 hours after surgery. Moreover, we used the similar dose of intrathecal bupivacaine and postoperative multimodal analgesia protocol in all patients. Therefore, these factors should have caused minimal to no conflicts in this study. The serial postoperative TUG tests and the quadriceps strength tests from postoperative day 2 were significantly worse than those of preoperative period. These findings are in agreement with several previous studies ${ }^{27-29)}$, in which performance-based measures after TKA were impaired for a minimum of 6 weeks postoperatively until they gradually returned to show significant improvement.

Our study has some limitations. Firstly, the present study injected $100 \mathrm{~mL}$ of LIA mixture in group $\mathrm{A}+\mathrm{L}$, which seemed to be less than that in several studies. However, the overall amount of anesthetic agent and other cocktail agents were calculated based on patient's body weight and proper doses. Therefore, we limited only the total volume of the cocktail mixture. The 100$\mathrm{mL}$ volume was also considered appropriate for most of our patients who had small statures and small knees, which resulted in significantly extended time for the first morphine pain rescue and a lower percentage of patients who required pain rescue medication. Lastly, the LIA mixture contained morphine which might have affected the delay in requiring rescue doses of morphine via PCA. However, morphine used in the LIA mixture in the present study was not different from that in previous several studies ${ }^{16,26-27,30)}$ which compared different anesthetic techniques in TKA and reported on morphine consumption and side effects for pain rescue as their outcomes.

\section{Conclusions}

Although combined LIA and single-dose ACB under simple spinal analgesia in TKA provided similarly low level of postoperative pain, early ambulation ability, quadriceps strength at all points of measurement, and total morphine consumption, compared to single-dose ACB alone, combined LIA and single-dose $\mathrm{ACB}$ significantly reduced and delayed the first request for rescue analgesia after surgery.

\section{Conflict of Interest}

No potential conflict of interest relevant to this article was reported.

\section{References}

1. Strassels SA, Chen C, Carr DB. Postoperative analgesia: economics, resource use, and patient satisfaction in an urban teaching hospital. Anesth Analg. 2002;94:130-7.

2. Wang $\mathrm{H}$, Boctor B, Verner J. The effect of single-injection femoral nerve block on rehabilitation and length of hospital stay after total knee replacement. Reg Anesth Pain Med. 2002;27:139-44.

3. Charous MT, Madison SJ, Suresh PJ, Sandhu NS, Loland VJ, Mariano ER, Donohue MC, Dutton PH, Ferguson EJ, Ilfeld BM. Continuous femoral nerve blocks: varying local anesthetic delivery method (bolus versus basal) to minimize quadriceps motor block while maintaining sensory block. Anesthesiology. 2011;115:774-81.

4. Jaeger P, Nielsen ZJ, Henningsen MH, Hilsted KL, Mathiesen O, Dahl JB. Adductor canal block versus femoral nerve block and quadriceps strength: a randomized, double-blind, placebo-controlled, crossover study in healthy volunteers. Anesthesiology. 2013;118:409-15.

5. Affas F, Nygards EB, Stiller CO, Wretenberg P, Olofsson C. Pain control after total knee arthroplasty: a randomized trial comparing local infiltration anesthesia and continuous femoral block. Acta Orthop. 2011;82:441-7.

6. Ilfeld BM. Continuous peripheral nerve blocks: a review of the published evidence. Anesth Analg. 2011;113:904-25.

7. Ilfeld BM, Duke KB, Donohue MC. The association between lower extremity continuous peripheral nerve blocks and patient falls after knee and hip arthroplasty. Anesth Analg. 2010;111:1552-4.

8. Jaeger P, Grevstad U, Henningsen MH, Gottschau B, Ma- 
thiesen O, Dahl JB. Effect of adductor-canal-blockade on established, severe post-operative pain after total knee arthroplasty: a randomised study. Acta Anaesthesiol Scand. 2012;56:1013-9.

9. Jenstrup MT, Jaeger P, Lund J, Fomsgaard JS, Bache S, Mathiesen O, Larsen TK, Dahl JB. Effects of adductor-canalblockade on pain and ambulation after total knee arthroplasty: a randomized study. Acta Anaesthesiol Scand. 2012; 56:357-64.

10. Lund J, Jenstrup MT, Jaeger P, Sorensen AM, Dahl JB. Continuous adductor-canal-blockade for adjuvant post-operative analgesia after major knee surgery: preliminary results. Acta Anaesthesiol Scand. 2011;55:14-9.

11. Burckett-St Laurant D, Peng P, Giron Arango L, Niazi AU, Chan VW, Agur A, Perlas A. The nerves of the adductor canal and the innervation of the knee: an anatomic study. Reg Anesth Pain Med. 2016;41:321-7.

12. Li D, Yang Z, Xie X, Zhao J, Kang P. Adductor canal block provides better performance after total knee arthroplasty compared with femoral nerve block: a systematic review and meta-analysis. Int Orthop. 2016;40:925-33.

13. Elkassabany NM, Antosh S, Ahmed M, Nelson C, Israelite C, Badiola I, Cai LF, Williams R, Hughes C, Mariano ER, Liu J. The risk of falls after total knee arthroplasty with the use of a femoral nerve block versus an adductor canal block: a double-blinded randomized controlled study. Anesth Analg. 2016;122:1696-703.

14. Bianconi M, Ferraro L, Traina GC, Zanoli G, Antonelli T, Guberti A, Ricci R, Massari L. Pharmacokinetics and efficacy of ropivacaine continuous wound instillation after joint replacement surgery. Br J Anaesth. 2003;91:830-5.

15. Kerr DR, Kohan L. Local infiltration analgesia: a technique for the control of acute postoperative pain following knee and hip surgery: a case study of 325 patients. Acta Orthop. 2008;79:174-83.

16. Busch CA, Shore BJ, Bhandari R, Ganapathy S, MacDonald SJ, Bourne RB, Rorabeck CH, McCalden RW. Efficacy of periarticular multimodal drug injection in total knee arthroplasty: a randomized trial. J Bone Joint Surg Am. 2006;88: 959-63.

17. Essving P, Axelsson K, Aberg E, Spännar H, Gupta A, Lundin A. Local infiltration analgesia versus intrathecal morphine for postoperative pain management after total knee arthroplasty: a randomized controlled trial. Anesth Analg. 2011;113:926-33.

18. Carli F, Clemente A, Asenjo JF, Kim DJ, Mistraletti G, Go- marasca M, Morabito A, Tanzer M. Analgesia and functional outcome after total knee arthroplasty: periarticular infiltration vs continuous femoral nerve block. Br J Anaesth. 2010; 105:185-95.

19. Toftdahl K, Nikolajsen L, Haraldsted V, Madsen F, Tonnesen EK, Soballe K. Comparison of peri- and intraarticular analgesia with femoral nerve block after total knee arthroplasty: a randomized clinical trial. Acta Orthop. 2007;78:172-9.

20. Koh IJ, Kang YG, Chang CB, Do SH, Seong SC, Kim TK. Does periarticular injection have additional pain relieving effects during contemporary multimodal pain control protocols for TKA?: a randomised, controlled study. Knee. 2012; 19:253-9.

21. Perlas A, Kirkham KR, Billing R, Tse C, Brull R, Gandhi R, Chan VW. The impact of analgesic modality on early ambulation following total knee arthroplasty. Reg Anesth Pain Med. 2013;38:334-9.

22. Andersen HL, Gyrn J, Møller L, Christensen B, Zaric D. Continuous saphenous nerve block as supplement to singledose local infiltration analgesia for postoperative pain management after total knee arthroplasty. Reg Anesth Pain Med. 2013;38:106-11.

23. Podsiadlo D, Richardson S. The timed "Up \& Go": a test of basic functional mobility for frail elderly persons. J Am Geriatr Soc. 1991;39:142-8.

24. Judd DL, Thomas AC, Dayton MR, Stevens-Lapsley JE. Strength and functional deficits in individuals with hip osteoarthritis compared to healthy, older adults. Disabil Rehabil. 2014;36:307-12.

25. Sawhney M, Mehdian H, Kashin B, Ip G, Bent M, Choy J, McPherson M, Bowry R. Pain after unilateral total knee arthroplasty: a prospective randomized controlled trial examining the analgesic effectiveness of a combined adductor canal peripheral nerve block with periarticular infiltration versus adductor canal nerve block alone versus periarticular infiltration alone. Anesth Analg. 2016;122:2040-6.

26. Spreng UJ, Dahl V, Hjall A, Fagerland MW, Ræder J. Highvolume local infiltration analgesia combined with intravenous or local ketorolac+morphine compared with epidural analgesia after total knee arthroplasty. Br J Anaesth. 2010; 105:675-82.

27. Reinhardt KR, Duggal S, Umunna BP, Reinhardt GA, Nam $\mathrm{D}$, Alexiades M, Cornell CN. Intraarticular analgesia versus epidural plus femoral nerve block after TKA: a randomized, double-blind trial. Clin Orthop Relat Res. 2014;472:1400-8.

28. Shah NA, Jain NP, Panchal KA. Adductor canal blockade 
following total knee arthroplasty-continuous or single shot technique? Role in postoperative analgesia, ambulation ability and early functional recovery: a randomized controlled trial. J Arthroplasty. 2015;30:1476-81.

29. Amarase C, Tanavalee A, Veerasethsiri P, Ngarmukos S. Should we use conventional or functional performance measures for evaluation of immediate outcomes after TKA? Thai
J Orthop Surg. 2016;40:3-10.

30. Nader A, Kendall MC, Manning DW, Beal M, Rahangdale R, Dekker R, De Oliveira GS Jr, Kamenetsky E, McCarthy RJ. Single-dose adductor canal block with local infiltrative analgesia compared with local infiltrate analgesia after total knee arthroplasty: a randomized, double-blind, placebocontrolled trial. Reg Anesth Pain Med. 2016;41:678-84. 